\title{
Dump system concepts for the Future Circular Collider
}

\author{
W. Bartmann, M. Atanasov, M. J. Barnes, J. Borburgh, F. Burkart, B. Goddard, T. Kramer, \\ A. Lechner, A. Sanz Ull, R. Schmidt, L. S. Stoel, R. Ostojic, J. Rodziewicz, and P. van Trappen \\ CERN, CH-1211 Geneva 23, Switzerland \\ D. Barna \\ WIGNER Research Centre for Physics, 1121 Budapest, Konkoly-Thege Miklós út 29-33, Hungary
}

(Received 25 November 2016; published 10 March 2017)

\begin{abstract}
The Future Circular Collider (FCC-hh) beam dump system must provide a safe and reliable extraction and dilution of the stored beam onto a dump absorber. Energy deposition studies show that damage limits of presently used absorber materials will already be reached for single bunches at $50 \mathrm{TeV}$. A fast field rise of the extraction kicker is required in order to sufficiently separate swept single bunches on the extraction protection absorbers in case of an asynchronous beam dump. In line with this demand is the proposal of a highly segmented extraction kicker system which allows for accepting a single kicker switch erratic and thus, significantly reduces the probability of an asynchronous beam dump. Superconducting septa are foreseen to limit the overall system length and power consumption. Two extraction system concepts are presented and evaluated regarding overall system length, energy deposition on absorbers, hardware requirements, radiation issues, and layout flexibility.
\end{abstract}

DOI: 10.1103/PhysRevAccelBeams.20.031001

\section{INTRODUCTION}

The Future Circular Collider (FCC-hh) dump systems have to abort the beam in a safe and reliable way at any energy level between injection and collision. The high beam rigidity and stored beam energy at collision energy, see Table I, pose a serious challenge for the extraction and dilution systems, respectively. Failure cases within the system which could lead to an asynchronous beam dump are of special concern and dominate the system design.

\section{A. Basic assumptions}

The dump system is considered to be triggered by an external signal from an interlock system. There is one dump system per beam which extracts the full beam in one turn onto an external dump block. In order to avoid an uncontrolled sweep of particles during the extraction kicker field rise, one or several abort gaps are considered in the filling pattern. Active and passive beam dilution is applied according to the damage limits of the dump block.

\section{EXTRACTION STRAIGHT BASELINE LAYOUT}

The ring layout considers two extended straight sections, each of $4.2 \mathrm{~km}$ length to house the extraction

Published by the American Physical Society under the terms of the Creative Commons Attribution 4.0 International license. Further distribution of this work must maintain attribution to the author(s) and the published article's title, journal citation, and DOI. and collimation systems. As a baseline, one extraction system per extended straight section is considered, Fig. 1. The betatron collimation system is located downstream of the extraction on the same beam, while the momentum collimation system is considered to be on the other beam. This configuration is critical with respect to loss showers from the momentum collimation system onto sensitive electronics equipment of the dump kickers. A careful study of shower attenuation by shielding or reconsidering the layout is required. A recently studied alternative layout features both extraction systems in one straight section. Why such a layout is preferable for the extraction systems is detailed in the following sections.

\section{A. Dump system layout and optics}

The suggested dump system concept is similar to the LHC design. The extraction kickers are deflecting the beam

TABLE I. Beam parameters for FCC injection and extraction.

\begin{tabular}{lccc}
\hline \hline Beam parameters & Unit & Injection & Extraction \\
\hline Kinetic energy & $\mathrm{TeV}$ & 3.3 & 50 \\
$\beta_{\text {rel }}$ & & $\approx 1$ & $\approx 1$ \\
$\gamma_{\text {rel }}$ & & 3518 & 53290 \\
Revolution period & $\mu \mathrm{s}$ & 333 & 333 \\
Magnetic rigidity & $\mathrm{T} . \mathrm{m}$ & 11011 & 166785 \\
\# bunches & & 10600 & 10600 \\
Bunch intensity & & $1 \times 10^{11}$ & $1 \times 10^{11}$ \\
Transverse emittances & $\mu \mathrm{m}$ & 2.2 & 2.2 \\
Total beam energy & $\mathrm{GJ}$ & 0.56 & 8.5 \\
\hline \hline
\end{tabular}




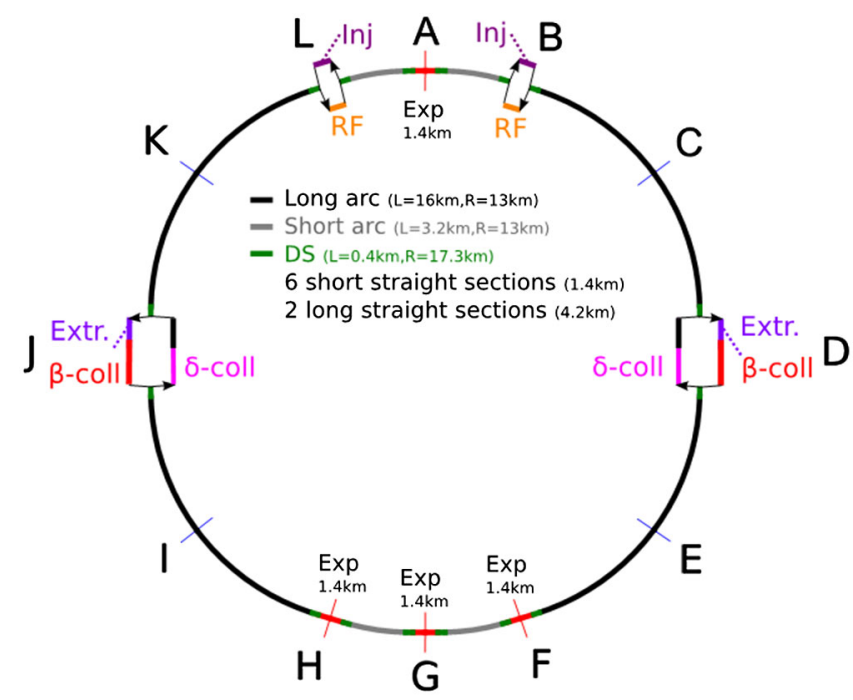

FIG. 1. Layout of the FCC collider. Courtesy D. Schulte.

in the horizontal plane to clear the blade of a Lambertson septum which is deflecting the beam in the vertical plane outside the cryostat of the downstream quadrupole Fig. 2. The robustness of Lambertson septa with respect to impact of radiation to coils is the main motivation of this design but novel designs of superconducting septa are being investigated which might require deflecting the beam in the same plane as does the kicker [1]. The most critical failure case is an erratic turn-on of the extraction kicker switches, which is not synchronized with the abort gap. In such a case some bunches will be swept over the aperture according to the rise time shape of the kickers. To protect the superconducting quadrupole and further machine elements downstream, one absorber is placed upstream of the first superconducting quadrupole, Fig. 2. The absorber needs to be $\sim 10 \mathrm{~m}$ long and a few $\mathrm{cm}$ wide to sufficiently attenuate the longitudinal and radial shower components. The longitudinal distance between kicker system and absorber is chosen such that in case of beam impact the energy deposition from the secondary particle shower is below the damage limit of the superconducting coils of the quadrupole. For the LHC, the damage limit of NbTi coils in case of fast beam losses is assumed to be around $100 \mathrm{~J} / \mathrm{cm}^{3}$ [2]. As a first estimate, it can be assumed that the limit is of the same order of magnitude for FCC magnet coils. To mitigate damage of the absorber itself, it is placed as far downstream from the kicker magnets as feasible in order to increase the bunch separation during the sweep. Losing the full beam on the same spot on the protection absorber would lead to hydrodynamic tunneling [3], and thus presents a not acceptable failure case. Table II shows the number of bunches, their separation and the rms beam size on the respective protection absorbers.

The transverse distance to the beam is defined by the hierarchy of the collimation system. Assuming a three stage cleaning system [4], the extraction protection absorber shall be placed transversely in the shadow of the secondary collimators in order not to deteriorate the cleaning efficiency and to minimize activation of the downstream area in standard operational conditions. On the other hand, this protection element has to shadow tertiary absorbers, which are made of high atomic number material and therefore have lower damage thresholds for primary particle impact [5]. Depending on the collimation system design and the energy, this position might be around 8 to 12 betatron $\sigma$ distance from the beam. Respecting the above-mentioned protection element hierarchy while taking into account tolerances for optics, orbit, setup errors and mechanical alignment is one of the limiting factors for the $\beta^{*}$ reach and consequently luminosity. In case of nonzero dispersion in the extraction region, its contribution has to be taken into account which will further limit luminosity performance. Aiming at a dispersion contribution of less than half a betatron $\sigma$ limits the maximum dispersion to about $40 \mathrm{~cm}$ in the extraction region.

The optics is adapted for mainly two reasons, beam dilution on the absorbers and minimum oscillation from a single kicker pre-fire, Fig. 3. High betatron functions in both planes (minimum of $800 \mathrm{~m}$ ) are aimed at the protection absorber to reach the required dilution for single bunch impact, see section below. A low betatron function in the kicker bending plane is required for the concept of accepting at maximum a $1 \sigma$ oscillation for up to one turn in case of a single kicker prefire [6]. For the dump system at LHC, a kicker prefire is detected internally and all the remaining kickers are retriggered within $800 \mathrm{~ns}$. For the FCC dump system it is being envisaged not to retrigger all the remaining kickers in case of a single unit prefire, but to wait until the beam is synchronized with the abort gap to trigger a dump without sweeping particles over the machine aperture. In order to keep the beam displacement from a single unit as small as possible, the kicker system is being

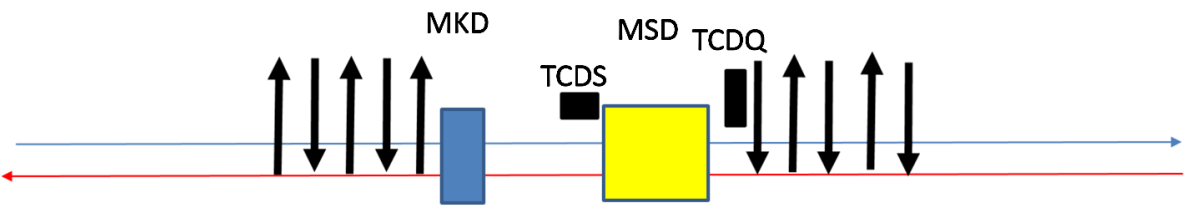

FIG. 2. Sketch of the dump concept. Arrows are indicating quadrupoles, MKD and MSD denote the extraction kicker and septum magnets, TCDS and TCDQ the protection absorbers for the septum and the quadrupoles, respectively. 
TABLE II. Beam parameters on the protection absorbers in the baseline design.

\begin{tabular}{lcccc}
\hline \hline & $\begin{array}{c}\# \\
\text { bunches }\end{array}$ & $\begin{array}{c}\text { Separation } \\
{[\mathrm{mm}]}\end{array}$ & $\begin{array}{c}\text { Beam size } \\
\mathrm{rms}[\mathrm{mm}]\end{array}$ & $\beta_{x} / \beta_{y}[\mathrm{~m}]$ \\
\hline $\begin{array}{c}\text { Septum } \\
\text { protection }\end{array}$ & 18 & $1.2-1.3$ & 0.20 & $970 / 970$ \\
$\begin{array}{c}\text { Quadrupole } \\
\text { protection }\end{array}$ & 5 & 2 & 0.34 & $2790 / 2790$ \\
\hline \hline
\end{tabular}

designed with high segmentation of about 300 units and the betatron function in the bending plane is minimized [7]. The kicker system has to reach its field within $1 \mu \mathrm{s}$ in order to provide sufficient bunch separation on the absorbers. Thus, the betatron function in the nonbending plane is also minimized to lower the system inductance. To reduce the time between a prefire and synchronization with the abort gap, it is beneficial to foresee several abort gaps in the machine.

The feasibility of this concept has to be studied with respect to load on the collimation system and beam-beam effects. This scenario should be compared to other failure scenarios leading to a sudden beam orbit excursion which should define the maximum acceptable oscillation amplitude. Until there is a common aperture strategy available for the FCC collider, all the hardware elements are designed for $\pm 10 \sigma \pm 4 \mathrm{~mm}$ beam-stay-clear area which is guided by LHC experience. The $4 \mathrm{~mm}$ accounts for orbit offsets, alignment tolerances, and optics uncertainties. The required kicker and septum parameters of this concept are summarized in Table III. The full length of this single extraction system between dispersion suppressors is $2.2 \mathrm{~km}$. The handover to the betatron collimation system is not yet optimized in this optics.

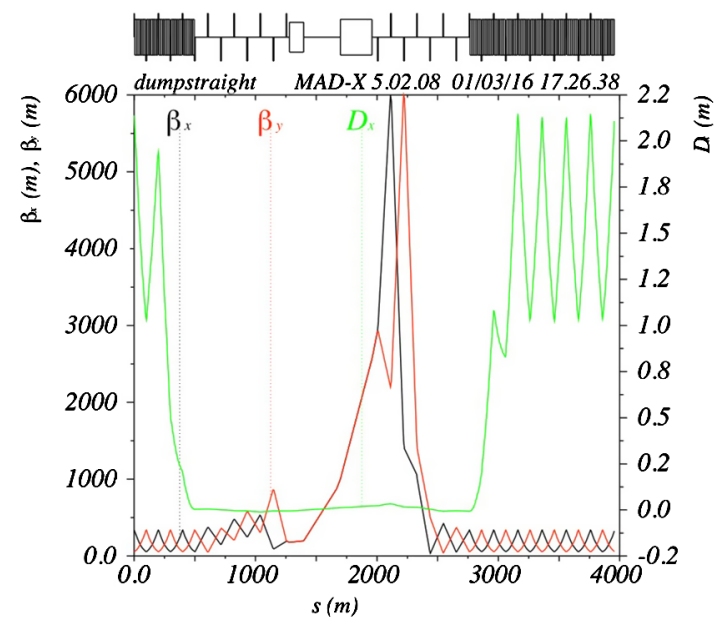

FIG. 3. Layout and optics of the dump concept. The dipole at the left end of the long central drift is the extraction kicker, followed by the extraction septum.
TABLE III. Parameters of kicker and septum for FCC extraction for the baseline concept.

\begin{tabular}{lccc}
\hline \hline Hardware parameters & Unit & Kicker & Septum \\
\hline Deflection & mrad & 0.13 & 3.1 \\
Integrated field & T.m & 22 & 513 \\
Available system length & $\mathrm{m}$ & 120 & 245 \\
Effective septum thickness & $\mathrm{mm}$ & $\ldots$ & 25 \\
Maximum leak field & & $\ldots$ & $3 \times 10^{-4}$ \\
Rise time & $\mu \mathrm{s}$ & 1 & $\ldots$ \\
Flattop length & $\mu \mathrm{s}$ & $\geq 333$ & $\geq 333$ \\
Flattop stability & $\%$ & \pm 5 & \pm 1 \\
GFR h/v & $\mathrm{mm}$ & $15 / 15$ & $33 / 33$ \\
\hline \hline
\end{tabular}

\section{B. Optional extraction bump and cryostat passage}

There are several options to ease the extraction elements requirements. Adding a bump which moves the beam close the septum blade can be used to reduce the kicker deflection. This bump would need a ramp program to be off at injection energy where the beam size is largest, and ramping up to its maximum at collision energy to reduce the kick strength when the beam is most rigid but also smallest in size.

The septum strength can be significantly reduced if the beam does not need to pass outside the downstream quadrupole but can be led through an opening in the cryostat. The downstream drift can be used to place further dipole magnets to direct the extracted beam into the dump channel.

Both options require additional hardware, for the bumper system and its powering, or a new hardware design of presently foreseen elements like the quadrupole with an extra opening in the cryostat for beam passage.

\section{PROTECTION ABSORBERS}

The energy deposited by $50 \mathrm{TeV}$ proton bunches in absorber materials poses a severe challenge for the robustness of protection devices. The maximum energy deposition density and temperature inside an absorber are governed by electromagnetic showers which are concentrated along the shower core. Electromagnetic showers are mainly induced by the decay products ( $\gamma$-pairs) of $\pi^{0}$-mesons produced in hadronic cascades. The $\pi^{0} \mathrm{~s}$ carry on average about one third of the energy released in hadronnucleus collisions. Compared to the LHC, the energy deposition density in absorber materials increases due to the higher proton energy and the smaller angular opening of showers.

Depending on the transverse bunch size $\left(\sigma_{x} \times \sigma_{y}\right)$, and hence the $\beta_{x^{-}}$and $\beta_{y}$-functions at the absorber location, already a single $50 \mathrm{TeV}$ FCC bunch can damage some of the most robust absorber materials like Graphite or carbonfiber reinforced carbon (CfC) presently used for intercepting high-energy particle beams. The stresses generated 
by the thermal shock can compromise the integrity of such materials already well below their maximum service temperature, which is typically around $3000^{\circ} \mathrm{C}$ for Graphite or $\mathrm{CfC}$ in vacuum. In order to safely absorb FCC bunches swept across the aperture in case of an extraction accident, the bunch size needs to be sufficiently large and bunches need to be sufficiently diluted across the absorber front face. In the following, some constraints for the extraction region optics are derived assuming that the robustness of materials is similar to those used for LHC absorbers.

Figure 4 shows the maximum energy deposition density and temperature, as a function of $\sqrt{\beta_{x} \beta_{y}}$, induced by a single FCC bunch in a CfC composite with a density of
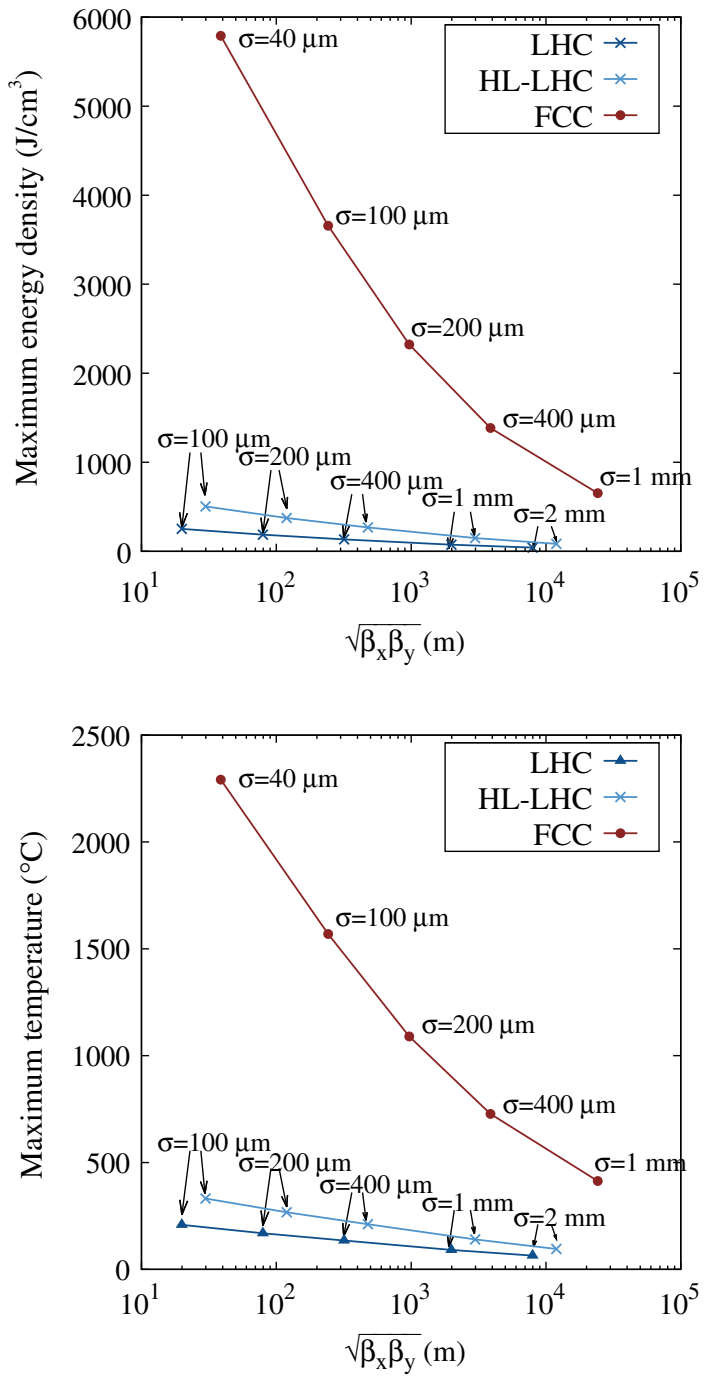

FIG. 4. Maximum energy deposition density (top) and temperature (bottom) versus $\sqrt{\beta_{x} \beta_{y}}$, induced by a single LHC, HL-LHC and FCC proton bunch in a $\mathrm{CfC}$ absorber with a density of $1.4 \mathrm{~g} / \mathrm{cm}^{3}$. The energy densities were obtained with FLUKA simulations, assuming that beams are round $\left(\beta_{x}=\beta_{y}\right)$. The physical beam size $\left(\sigma=\sigma_{x, y}\right)$ is displayed next to the data points. The FCC bunch parameters are specified in Table I. The LHC and HL-LHC beam parameters are detailed in the text.
$1.4 \mathrm{~g} / \mathrm{cm}^{3}$. The contribution of the dispersive component to the beam size is neglected, i.e. $\sigma_{x, y}=\sqrt{\varepsilon \beta_{x, y} / \beta \gamma}$, where $\varepsilon$ is the transverse normalized emittance and $\beta \gamma$ is the product of the relativistic factors. The emittance and bunch intensity were taken from Table I. The results were derived with particle shower simulations using the FLUKA Monte Carlo code [8,9]. For comparison, the figure also shows the peak energy deposition density and temperature induced by a nominal $7 \mathrm{TeV}$ LHC (HL-LHC) bunch, with a normalized transverse emittance of $3.5(2.1) \mu \mathrm{m} \cdot \mathrm{rad}$ and a bunch intensity of $1.15(2.2) \times 10^{11}$ protons. For the same $\beta$-functions, a FCC bunch gives rise to $25-27$ times higher peak energy densities than a LHC bunch, and 10-12 times higher energy densities than a HL-LHC bunch. Depending on the bunch size and beam energy, the maximum energy deposition and hence the hottest spot occurs at different depths between 1.5 and $3 \mathrm{~m}$ inside the absorber. To illustrate the effect of the beam energy, Fig. 5 compares two-dimensional energy deposition density maps for a $7 \mathrm{TeV}$ and a $50 \mathrm{TeV}$ bunch, assuming that the physical beam size $(\sigma)$ is the same. The depth at which the energy deposition density is maximum differs by less than $1 \mathrm{~m}$ between the two beam energies.
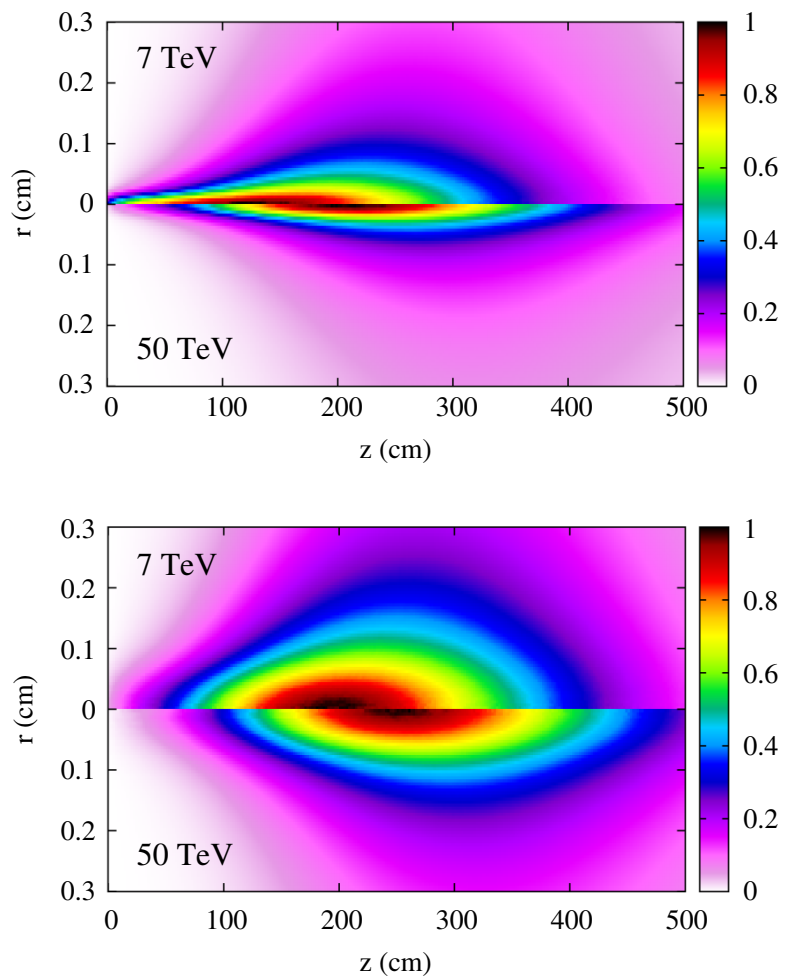

FIG. 5. Energy deposition density maps, calculated with FLUKA, for a $7 \mathrm{TeV}$ bunch (top half) and a $50 \mathrm{TeV}$ bunch (bottom half) in a CfC absorber with a density of $1.4 \mathrm{~g} / \mathrm{cm}^{3}$. The beam size $(\sigma)$ is assumed to be $100 \mu \mathrm{m}$ in the upper figure, and $400 \mu \mathrm{m}$ in the lower figure. The beam direction is from the left to the right. The $z$-variable indicates the absorber depth and the $r$-variable the radial distance from the beam axis. All maps are expressed as a fraction of the maximum energy deposition density. 
Composites as the one considered in Fig. 4 are the core material for several protection devices in the LHC extraction region [10]. They combine a good mechanical strength with a low density, which reduces the shower-induced peak load. As demonstrated in Fig. 4, the peak temperature induced by a FCC bunch exceeds $1000^{\circ} \mathrm{C}$ if $\sqrt{\beta_{x} \beta_{y}}$ is less than $\sim 1 \mathrm{~km}$, and reaches $1500^{\circ} \mathrm{C}$ if $\sqrt{\beta_{x} \beta_{y}}$ is less than $\sim 300 \mathrm{~m}$. Thermo-structural studies carried out for the LHC (see, e.g., [11]) showed that tensile stresses typically exceed the tensile strength of CfC composites used in LHC absorbers if beam-induced peak temperatures are as high as $1500^{\circ} \mathrm{C}$. These results indicate that $\beta$-functions at absorber locations need to be larger than several hundred meters in order to safely absorb a single FCC bunch in a LHC-like absorber.

If multiple bunches are swept across an absorber in case of an asynchronous beam dump, the induced energy deposition density and temperature are driven by the overlap of transverse shower profiles of individual bunches. In addition to the $\beta$-functions, the peak energy deposition density therefore depends on the number of bunches intercepted by the absorber and on their transverse separation during impact. For the layouts and system parameters presented in this paper, the maximum number of bunches intercepted by protection absorbers in the extraction region is less than 20 (see Table II). Figure 6 shows the peak temperature generated by multiple bunches impacting on a $\mathrm{CfC}$ absorber, assuming that $\sqrt{\beta_{x} \beta_{y}}$ is $\sim 1 \mathrm{~km}$, which is the minimum $\sqrt{\beta_{x} \beta_{y}}$ at absorbers given in Table II. The different curves in the figure show the effect of a different transverse bunch separation. In order to absorb 20 bunches and keep the maximum temperature around $1500^{\circ} \mathrm{C}$, neighboring bunches need to be

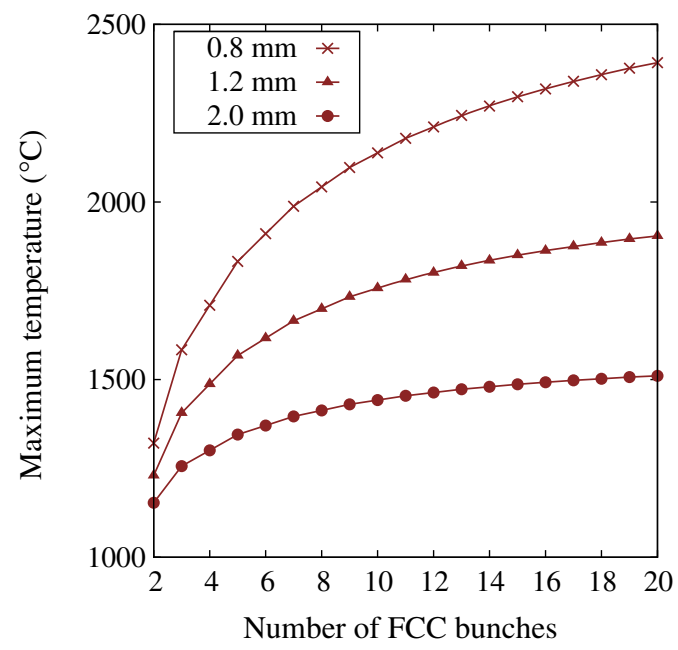

FIG. 6. Maximum temperature in a CfC absorber $\left(1.4 \mathrm{~g} / \mathrm{cm}^{3}\right)$ as a function of the number of FCC bunches swept across the absorber front face. The temperatures were calculated in adiabatic limit, using FLUKA energy deposition results. The transverse bunch size $(\sigma)$ was assumed to be $200 \mu \mathrm{m}$, which corresponds to $\sqrt{\beta_{x} \beta_{y}} \approx 1 \mathrm{~km}$. The different curves assume a different transverse bunch separation in the sweep direction. separated by about $2 \mathrm{~mm}$. The separation can be somewhat smaller if fewer bunches impact on the absorber or if $\sqrt{\beta_{x} \beta_{y}}$ is higher than the $1 \mathrm{~km}$ assumed in the figure.

The adopted temperature limit allows only for the first approximative assessment of constraints arising from a limited absorber robustness. Thermo-structural studies are necessary to refine these constraints based on the stresses generated by the beam-induced temperature gradients inside the absorbers. In addition, the derived limits are based upon the assumption that similar carbon composites as in the LHC are used. Some gain might be achievable with other materials (e.g. Graphite or carbon composites with lower density to reduce further the peak temperature). Robustness to beam impact is however not the only criterium for selecting absorber materials. They must also exhibit a low outgassing rate to meet vacuum specifications, and they should have a good electrical conductivity to minimize the resistive wall heating of the protection device.

Apart from being robust enough, a protection device needs to shield sufficiently downstream equipment such as septa and magnets from the showers generated by the $50 \mathrm{TeV}$ protons. For a $\mathrm{CfC}$ absorber, as the one considered above, the shower maximum occurs in a depth of roughly $3 \mathrm{~m}$. To optimize the absorption of secondary showers, other materials, like higher-density graphite, can be used further downstream where the shower-induced energy deposition density is lower. Similar sandwich solutions have been adopted for the LHC [10]. In case of the FCC, a total absorber length of the order of $10 \mathrm{~m}$ is sufficient to protect equipment components which are in the direct geometrical shadow of the absorber. FLUKA shower simulations indicate that for the worst case in Table II, where 18 bunches are swept across the septum protection absorber, the temperature increase in the steel septum blade is less than $30 \mathrm{~K}$ if a $9 \mathrm{~m}$ long LHC-like absorber sandwich of low- and high-density CfC is used.

If mis-steered FCC bunches impact close to the absorber edge or if they graze along the absorber, the energy deposition in downstream equipment is dominated by secondary particles leaking through the absorber gap. For such accident scenarios, one can only achieve a limited gain by further increasing the absorber length and one needs in addition masklike shower absorbers just upstream of the magnet or septum to protect components which are not in the direct geometrical shade of the primary absorber.

\section{EXTRACTION STRAIGHT ALTERNATIVE LAYOUT}

Iterations have been undertaken to optimize the straight sections layout, their lengths, and the distribution of experiments and technical systems into these straights. As an outcome, a straight section which combines the extractions for both beams is favored.

Within studying this possibility of combining both extractions in a single straight, results from energy deposition studies for FCC on protection absorbers were taken 


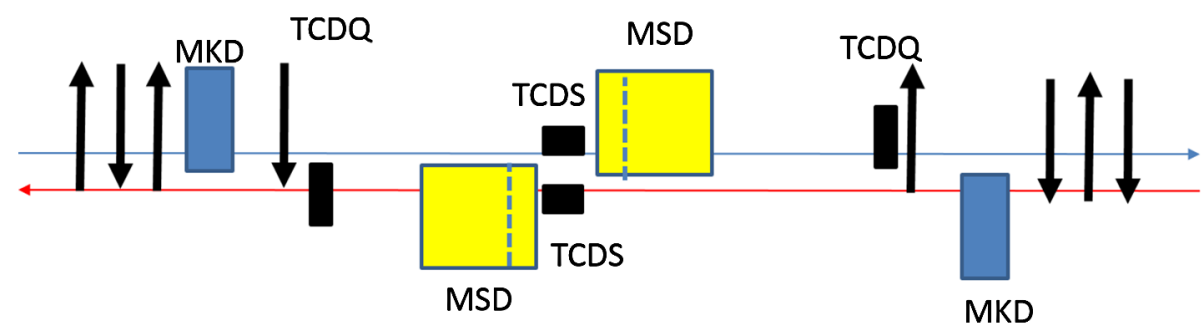

FIG. 7. Layout of the dump concept. Arrows are indicating quadrupoles, MKD and MSD denote the extraction kicker and septum magnets, TCDS and TCDQ the protection absorbers for the septum and the quadrupoles, respectively.

into account. In the baseline design, drifts downstream the extraction area are reserved to place secondary absorbers of higher atomic number material for better attenuation of particle showers. The FCC energy deposition calculations presented in the section above show however, that sufficient attenuation can be provided with a single stage absorber system combined with fixed aperture masks to avoid damage of the superconducting coils of the downstream quadrupoles. Therefore, the two extraction systems could be overlaid as much as possible by omitting the quadrupole section in between the septa, Fig. 7. The symmetry point of the dump systems is given by the septum protection absorbers which are most prone to damage in case of an asynchronous beam dump due to their proximity to the dump kickers. In order to sufficiently dilute the beam, the betatron functions in both planes amount to more than $1 \mathrm{~km}$. At the quadrupole protection absorber the beam is strongly diluted in the horizontal plane with a betatron function of $3.3 \mathrm{~km}$, less diluted in the vertical plane with a betatron function of $260 \mathrm{~m}$, Fig. 8. Compared to the baseline scheme, this layout profits from a strong defocusing quadrupole between kicker and septum. The additional kick enhances bunch separation on the septum protection to about $2 \mathrm{~mm}$ with a total load of 12 bunches, Table IV. The quadrupole protection is impacted by 6 bunches. The load on the

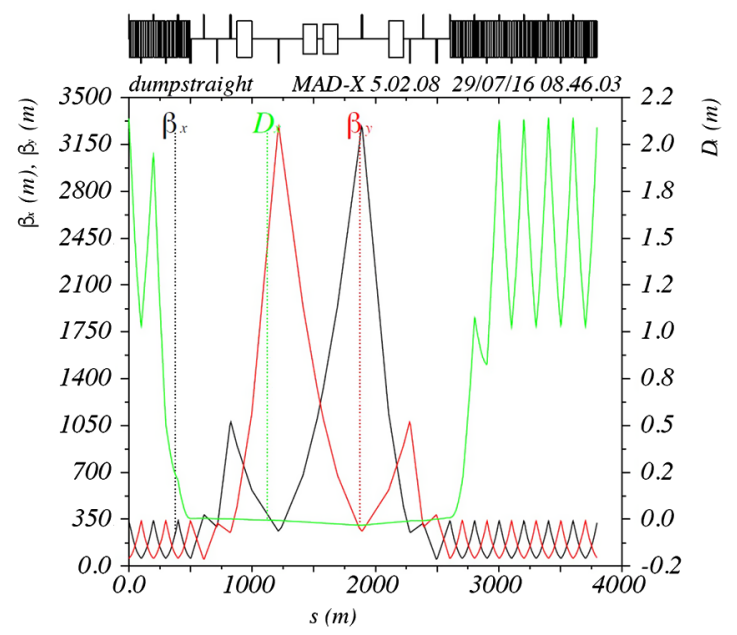

FIG. 8. Layout and optics of the alternative dump concept. The dipoles in the middle represent the septa, the elements to the outside of the straight the extraction kickers. absorbers for this scenario requires detailed studies of energy deposition. Considering however the temperature criterion adopted in the previous section and the results shown in Fig. 6, it seems probable that a particle sweep at full energy can be survived without damage. The septum deflection is reduced from $3 \mathrm{mrad}$ to $2 \mathrm{mrad}$ with respect to the baseline scheme due to a longer distance to the downstream quadrupole. For the septa it is foreseen to use about $20 \mathrm{~m}$ of normal conducting units at a moderate field level of $1.3 \mathrm{~T}$ to achieve a larger beam separation and at the same time shielding, both of which are required for the superconducting units downstream [12]. Superconducting septa are deployed to reach beam passage outside the cryostat of the downstream quadrupole. To reduce the probability of quenching these septa during extraction, massless superconducting shields which use persistent currents to expel an external field are being investigated [1]. The full length of the alternative extraction layout between dispersion suppressors is $2.1 \mathrm{~km}$. The required kicker and septum parameters are summarized in Table V.

TABLE IV. Beam parameters on the protection absorbers in the alternative design.

\begin{tabular}{lcccc}
\hline \hline & $\begin{array}{c}\# \\
\text { bunches }\end{array}$ & $\begin{array}{c}\text { Separation } \\
{[\mathrm{mm}]}\end{array}$ & $\begin{array}{c}\text { Beam size } \\
\text { rms }[\mathrm{mm}]\end{array}$ & $\beta_{x} / \beta_{y}[\mathrm{~m}]$ \\
\hline $\begin{array}{c}\text { Septum } \\
\text { protection }\end{array}$ & 12 & $1.9-2.1$ & $0.23 / 0.21$ & $1304 / 1123$ \\
$\begin{array}{c}\text { Quadrupole } \\
\text { protection }\end{array}$ & 6 & 2 & $0.36 / 0.10$ & $3285 / 264$ \\
\hline \hline
\end{tabular}

TABLE V. Parameters of kicker and septum at FCC extraction for the alternative concept.

\begin{tabular}{lccc}
\hline \hline Hardware parameters & Unit & Kicker & Septum \\
\hline Deflection & $\mathrm{mrad}$ & 0.06 & 2.2 \\
Integrated field & T.m & 10 & 370 \\
Available system length & $\mathrm{m}$ & 120 & 120 \\
Effective septum thickness & $\mathrm{mm}$ & $\ldots$ & 25 \\
Maximum leak field & & $\ldots$ & $3 \times 10^{-4}$ \\
Rise time & $\mu \mathrm{s}$ & 1 & $\ldots$ \\
Flattop length & $\mu \mathrm{s}$ & $\geq 333$ & $\geq 333$ \\
Flattop stability & $\%$ & \pm 5 & \pm 1 \\
GFR h/v & $\mathrm{mm}$ & $38 / 25$ & $28 / 19$ \\
\hline \hline
\end{tabular}




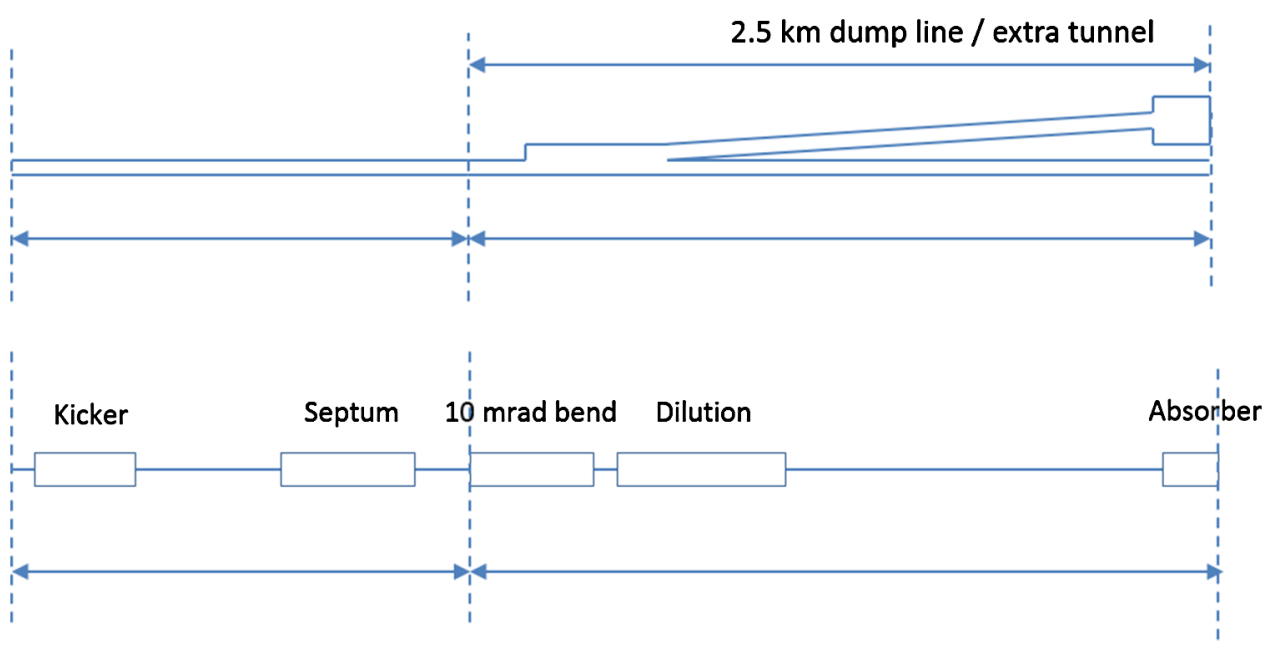

FIG. 9. Sketch of a dump tunnel separated from the arc.

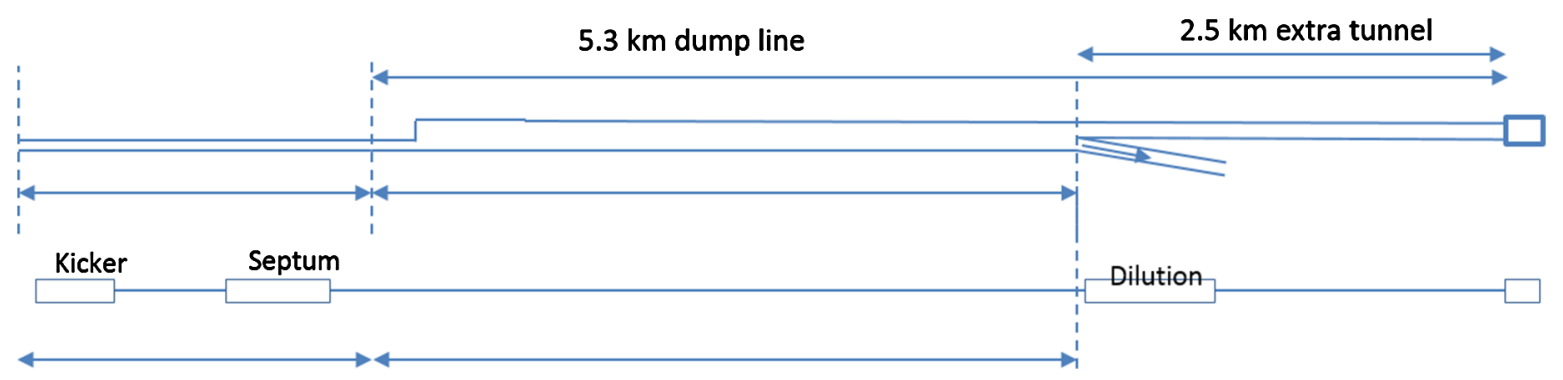

FIG. 10. Sketch of a dump tunnel downstream the collimation system.

\section{DUMP LINE LAYOUT AND DILUTION}

The dump line layout is dominated by the optimal dilution pattern on the dump block which is described in [13]. In the baseline layout the extraction system is followed by the betatron collimation system on the same beam. Placing the dilution kickers directly next to the collimation system is excluded for reasons of radiation to electronics and maintenance. To avoid this, the dump line can be guided into a separate tunnel which provides the necessary shielding. This requires a deflection equivalent to that of about one arc cell, see Fig. 9, and an extra tunnel of about $2.5 \mathrm{~km}$ length. The other option is to place the dilution kickers only after the collimation system. Here the extra bending is avoided since separation is provided by the collider arc, but still an additional tunnel of about $2.5 \mathrm{~km}$ length is needed from the end of the straight section, Fig. 10. In case of the alternative extraction concept the dilution kickers can be placed as soon as sufficient separation is given from the circulating beam elements. Radiation to electronics from protection absorbers is of a much lesser concern than that from the collimation system since they are in the shadow of the primary and secondary collimators for the circulating beam and in case of a severe impact during extraction, the system has already been triggered.

\section{CONCLUSIONS}

The FCC baseline layout considers extraction and collimation systems in one straight section which requires careful studies of radiation impact to electronics. This single extraction concept is dominated by protection absorber limits for single bunch dilution and bunch separation.

Recent iterations of the layout favor both extractions in a single straight section which avoids the radiation to electronics issue. Results of energy deposition studies show that a single stage extraction absorber provides sufficient attenuation of particle showers during an asynchronous beam dump to keep the peak energy deposition density below the damage level in the superconducting quadrupole coils. This result allows us to further optimize the extraction design incorporating both extractions in one straight.

The alternative scheme uses the septum protection absorbers as symmetry point and allows us to lower the requirements on kicker and septum while keeping the absorber limitations.

Deployment of an extraction bump and beam passage through the cryostat can ease the extraction hardware specifications at the expense of introducing new hardware.

The dump line layout for the baseline option requires either bending into a separate tunnel or placing the dilution kickers only after the arc bending starts to avoid 
radiation to electronics issues. For the alternative concept the dilution kickers can be placed next to the circulating beam elements.

The alternative concept is advantageous compared to the baseline scheme with respect to total length, hardware specifications and radiation to electronics concerns.

[1] D. Barna, in FCC Week 2016, Rome (2016), http://indico .cern.ch/event/438866/contributions/1085053/attachments/ 1256966/1856612/FCCw16-septum-Daniel-Barna.pdf.

[2] O. Bruning and J. Jeanneret, CERN Report No. LHCProject-Note-141, 1998.

[3] N. Tahir, F. Burkart, R. Schmidt, A. Shutov, D. Wollmann, and A. Piriz, Beam induced hydrodynamic tunneling in the future circular collider components, Phys. Rev. Accel. Beams 19, 081002 (2016).

[4] O. S. Bruning, P. Collier, P. Lebrun, S. Myers, R. Ostojic, J. Poole, and P. Proudlock, LHC Design Report (CERN, Geneva, 2004), Report No. CERN-2004-003-V-1, p. 548, https://cds.cern.ch/record/782076.

[5] M. Cauchi, O. Aberle, R.W. Assmann, A. Bertarelli, F. Carra, K. Cornelis, A. Dallocchio, D. Deboy, L. Lari, S. Redaelli et al., High energy beam impact tests on a LHC tertiary collimator at the CERN high-radiation to materials facility, Phys. Rev. ST Accel. Beams 17, 021004 (2014).

[6] B. Goddard et al., FCC Week 2016, Rome (2016), http://indico.cern.ch/event/438866/contributions/1085060/ attachments/1256481/1855033/FCC_week_Rome_2016_ asynch_dump2.pptx.

[7] T. Kramer et al., in FCC Week 2016, Rome (2016), http://indico.cern.ch/event/438866/contributions/1085005/ attachments/1256955/1855975/Kicker_systems_FCC_ week_2016.pptx.

[8] A. F. A. Ferrari, P. R. Sala, and J. Ranft, Reports No. CERN-2005-10, No. INFN/TC_05/11, No. SLACR-773, 2005.

[9] T. T. Böhlen et al., The FLUKA Code: Developments and Challenges for High Energy and Medical Applications, Nucl. Data Sheets 120, 211 (2014).

[10] M. G. Atanasov et al., in Proceedings of the 6th International Particle Accelerator Conference (2015).

[11] J. Uythoven et al., in Proceedings of the 5th International Particle Accelerator Conference (2014).

[12] A. Sanz Ull et al., FCC Week 2016, Rome (2016), http://indico.cern.ch/event/438866/contributions/1084992/ attachments/1256989/1856039/FCC_beam_dump_septa_ technologies_and_topologies.pptx.

[13] D. Barna et al., FCC Week 2016, Rome (2016). 